\title{
Impactos da COVID-19 nos cuidados de enfermagem no ambulatório oncológico de
}

\section{cabeça e pescoço}

Impacts of COVID-19 on nursing care in the head and neck oncology outpatient clinic

Impactos de la COVID-19 en el cuidado de enfermería en el ambulatorio de oncología de cabeza y cuello

Recebido: 30/01/2022 | Revisado: 05/02/2022 | Aceito: 11/02/2022 | Publicado: 17/02/2022

Lucimere Maria dos Santos

ORCID: https://orcid.org/0000-0003-3455-1268

Instituto Nacional de Câncer, Brasil

E-mail: luciemreuff@gmail.com

Marcia Santos

ORCID: https://orcid.org/0000-0002-3067-2495 Instituto Nacional de Câncer, Brasil E-mail: frija27@gmail.com

Teresa Cristina Silva Palermo

ORCID: https://orcid.org/0000-0001-8413-0336

Instituto Nacional de Câncer

E-mail: tpalermo@inca.gov.br

Luciana Nogueira dos Santos

ORCID: https://orcid.org/0000-0001-8303-3809

Instituto Nacional de Câncer, Brasil

E-mail: bioln.santos@gmail.com

Cristiane dos Santos Mendonça da Silva

ORCID: https://orcid.org/0000-0001-5699-2887

Instituto Nacional de Câncer, Brasil

E-mail: silva_cristiane100@yahoo.com.br

Aureonice Marinho

ORCID: https://orcid.org/0000-0001-6558-5917

Instituto Nacional de Câncer, Brasil

E-mail: aureonicemarinho@gmail.com

Julia Meireles da Costa

ORCID: https://orcid.org/0000-0002-5679-7683 Instituto Nacional de Câncer, Brasil

E-mail: meirelesjulia72@yahoo.com.br

Aline Peragene

ORCID: https://orcid.org/0000-0002-1462-9788 Instituto Nacional de Câncer, Brasil

E-mail: aperagene@gmail.com

\begin{abstract}
Resumo
Este estudo tem como objetivo analisar os impactos da COVID-19 no atendimento do paciente oncológico no ambulatório de cirurgia de cabeça e pescoço, por meio dos procedimentos realizados nos anos de 2019, 2020 e 2021. Método: Estudo qualitativo, descritivo sobre os procedimentos realizados pela equipe de enfermagem em um ambulatório de cirurgia de cabeça e pescoço. Os dados foram coletados no período de janeiro de 2019 a dezembro de 2021 e computados em planilha de Excel. Resultados: Mostraram a disparidade negativa no quantitativo de atendimentos ao paciente no ambulatório de cirurgia de cabeça e pescoço, no ano de 2019 em relação a 2020, em que ocorreu uma queda de 33\%. Comparando os resultados do ano de 2019 com 2021 percebeu-se uma queda de $25 \%$. Porém, quando comparado o ano de 2020 a 2021, notou-se um aumento de $12 \%$. No quantitativo de procedimentos realizados em 2020 houve uma queda de $31 \%$ em relação a 2019 e, em comparação a 2021, houve uma queda de $26 \%$, porém no ano de 2020 para 2021 houve um aumento de 13\%. Conclusão: A pandemia por COVID-19 gerou um forte impacto na assistência prestada aos pacientes do ambulatório de cirurgia de cabeça e pescoço, levando a uma redução do atendimento e da prestação dos cuidados de enfermagem a essa clientela, porém no início do cenário pandêmico as instituições tiveram que redesenhar os protocolos de cuidados para o enfrentamento da situação atual do país e do mundo, promovendo assim uma assistência mais segura e eficiente.
\end{abstract}

Palavras-chave: Cuidados de Enfermagem; Câncer de cabeça e pescoço; Covid-19. 


\begin{abstract}
This study aims to analyze the impacts of COVID-19 on cancer patient care in the head and neck surgery outpatient clinic, through the procedures performed in the years 2019, 2020 and 2021. Method: Qualitative, descriptive study on the procedures performed by the nursing team in a head and neck surgery outpatient clinic. Data were collected from January 2019 to December 2021 and computed in an Excel spreadsheet. Results: They showed the negative disparity in the number of patient visits in the head and neck surgery outpatient clinic, in 2019 compared to 2020, in which there was a $33 \%$ drop. Comparing the results of the year 2019 with 2021 , a drop of $25 \%$ was noticed. However, when comparing the year 2020 to 2021, there was an increase of $12 \%$. In the number of procedures performed in 2020 there was a $31 \%$ drop compared to 2019 and, compared to 2021, there was a 26\% drop, but in the year 2020 to 2021 there was an increase of $13 \%$. Conclusion: The COVID-19 pandemic had a strong impact on the care provided to patients at the head and neck surgery outpatient clinic, leading to a reduction in care and provision of nursing care to this clientele, but at the beginning of the pandemic scenario, institutions had to redesign care protocols to face the current situation in the country and in the world, thus promoting safer and more efficient care.
\end{abstract}

Keywords: Nursing care; Head and neck cancer; Covid-19.

\title{
Resumen
}

Este estudio tiene como objetivo analizar los impactos del COVID-19 en la atención al paciente oncológico en el ambulatorio de cirugía de cabeza y cuello, a través de los procedimientos realizados en los años 2019, 2020 y 2021. Método: Estudio descriptivo cualitativo sobre los procedimientos realizados por el equipo de enfermería en un ambulatorio de cirugía de cabeza y cuello. Los datos se recopilaron desde enero de 2019 hasta diciembre de 2021 y se calcularon en una hoja de cálculo de Excel. Resultados: Mostraron la disparidad negativa en el número de visitas de pacientes en la consulta externa de cirugía de cabeza y cuello, en 2019 en comparación con 2020, en el que hubo una caída del 33\%. Comparando los resultados del año 2019 con el 2021, se notó una caída del 25\%. Sin embargo, al comparar el año 2020 con el 2021, hubo un aumento del 12\%. En el número de procedimientos realizados en el 2020 hubo una caída del $31 \%$ con respecto al 2019 y con respecto al 2021 hubo una caída del 26\%, pero en el año 2020 al 2021 hubo un aumento del 13\%. Conclusión: La pandemia de COVID-19 tuvo un fuerte impacto en la atención a los pacientes en el ambulatorio de cirugía de cabeza y cuello, lo que provocó una reducción en la atención y prestación de cuidados de enfermería a esta clientela, pero al inicio del escenario de pandemia, las instituciones tenían rediseñar los protocolos de atención para enfrentar la situación actual del país y del mundo, promoviendo así una atención más segura y eficiente.

Palabras clave: Cuidado de enfermera; Cáncer de cabeza y cuello; Covid-19.

\section{Introdução}

A incidência e a mortalidade por câncer vêm aumentando no mundo, tornando-se os principais problemas de saúde pública, em parte pelo envelhecimento, pelo crescimento populacional, como também pela mudança na distribuição e na prevalência dos fatores de risco de câncer, especialmente aos associados ao desenvolvimento socioeconômico (Lagergren et al., 2016).

Segundo o Instituto Nacional de Câncer (INCA), para cada ano do triênio 2020/2022, o Brasil deverá registrar 624 mil casos novos de câncer. Dentre os casos de câncer, os tumores na região de Cabeça e Pescoço (CP) apresentam grande incidência e alta mortalidade. Na região da cavidade oral será de 11.180 casos em homens e de 4.010 em mulheres, já o câncer de tireoide será de 1.830 casos novos em homens e de 11.950 em mulheres (Brasil, 2019).

No que se refere aos casos de câncer de laringe esperados para o Brasil, será de 6.470 em homens e de 1.180 em mulheres. O tipo histológico mais frequente é o carcinoma epidermoide (CEC), que surge em mais de $90 \%$ dos casos (Brasil, 2019).

A cirurgia para remoção da lesão tumoral do paciente oncológico de cabeça e pescoço, bem como a realização da traqueostomia são os procedimentos mais comuns entre as modalidades de tratamentos discutidas pelos profissionais médicos devido às complicações respiratórias oriundas da progressão tumoral, comprometendo a autoimagem e a sua participação no convívio social (Mota et al., 2021).

O cuidado desenvolvido pela equipe de enfermagem no ambulatório de cirurgia da região de cabeça e pescoço se faz de forma individual e especializado, valorizando os contextos sociais, econômicos e culturais, aliados ao processo de promoção à saúde que deve acontecer de forma integrada com os indivíduos envolvidos nesse cenário, porém o mesmo sofreu 
interferência no que se refere ao acesso a essas ações no período da pandemia por COVID-19. Doença infecciosa causada pelo coronavírus SARS-COV-2 (WHO, 2020).

Destarte, foi possível perceber que a pandemia provocou a redução significativa do número de cirurgias para o tratamento do câncer, dos procedimentos de enfermagem realizados, do número de visitantes e acompanhantes, além da diminuição efetiva de profissionais por contaminação pelo SARS-COV-2, bem como a suspensão das reuniões e encontros entre pacientes, familiares e profissionais, que são de extrema relevância para a eficiência no tratamento, na orientação para o autocuidado, na promoção e recuperação da saúde e da autoestima dos pacientes de CP (Araújo et al., 2020).

A atual pandemia exigiu que os profissionais de saúde dedicados ao tratamento do câncer redesenhassem os cuidados oncológicos, atualizando de forma permanente a prática do cuidado, a fim de promover uma assistência segura e eficaz, mitigando assim os potenciais efeitos negativos da infecção pela COVID-19 em pacientes submetidos ao tratamento (Ramos, 2020).

Neste contexto, condições crônicas de doenças têm exigido dos indivíduos a utilização de tratamento em longo prazo, o uso de estratégias para o cuidado no domicílio, além da reestruturação de estilos de vida, como forma de cuidar de si. Sendo assim, os cuidados de enfermagem fornecidos aos pacientes de cabeça e pescoço são concebidos como uma experiência complexa e impactante, capaz de interferir no cuidar e na interação entre profissional e paciente.

Para além dos aspectos técnicos e emocionais, a enfermagem exercita a sua capacidade de superação na realização dos processos de cuidados ao paciente atendido no ambulatório de cirurgia de $\mathrm{CP}$, por meio de um dos atributos mais caros a este profissional: o reconhecimento da condição humana marcada pela fortuidade da vida em uma tomada de consciência acerca da singularidade constituinte de todo indivíduo (Santos, Santos \& Silva, 2016).

Desta forma, esse estudo tem como objetivo analisar os impactos da COVID-19 no atendimento do paciente oncológico no ambulatório de cirurgia de cabeça e pescoço, por meio dos procedimentos realizados nos anos de 2019, 2020 e 2021.

\section{Metodologia}

Estudo descritivo de abordagem qualitativa sobre os procedimentos realizados pela equipe de enfermagem em um ambulatório de cirurgia de cabeça e pescoço de um Hospital Federal, localizado na cidade do Rio de Janeiro/RJ. Os dados primários foram coletados no período de janeiro de 2019 a dezembro de 2021 e se deu por meio de pesquisa documental utilizando instrumento estabelecido pela instituição em todos os ambulatórios, onde os resultados são inseridos diariamente pela equipe de enfermagem. Estes dados são provenientes do livro de procedimentos de enfermagem. Foi utilizado o método de análise de conteúdo, visando organizar os dados e analisar os resultados obtidos. Os dados foram computados em planilha de Excel (Bardin, 2004).

\section{Resultados}

Os resultados apresentados demonstram as consequências das infecções provenientes da COVID-19, afetando os pacientes em tratamento para o câncer de forma mais severa que os outros. Sendo assim, alguns tipos de tratamentos clínicos e cirúrgicos programados na instituição foram cancelados ou adiados devido à priorização de leitos hospitalares para tratamento dos pacientes com COVID-19. Essa situação afetou diretamente o tratamento e o cuidado aos pacientes com câncer da região de cabeça e pescoço, que necessitavam de consultas de primeira vez para traçar o plano de tratamento o mais precoce possível ou de consultas subsequentes para continuidade do cuidado, já que se trata de um tipo de câncer de rápida evolução.

Os resultados demonstrados no gráfico a seguir, apresentam uma disparidade negativa no quantitativo de atendimentos ao paciente no ambulatório de cirurgia de cabeça e pescoço, no ano de 2019 em relação a 2020, ano em que 
ocorreu uma queda de 33\%. Comparando os resultados do ano de 2019 com 2021, percebeu-se uma queda de 25\%. Porém, quando comparado o ano de 2020 a 2021, nos atendimentos realizados a esta clientela, notou-se um aumento de $12 \%$.

Gráfico 1 - Pacientes atendidos pela equipe de enfermagem no ambulatório de cirurgia de cabeça e pescoço em 2019, 2020 e 2021.

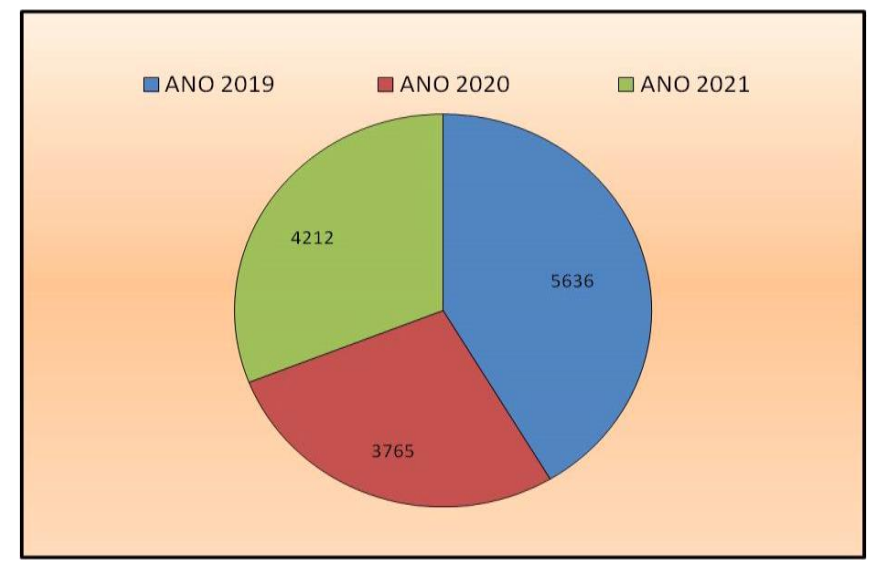

Fonte: INCA - HC1.

No gráfico acima se percebe que no ano de 2020 ocorreu uma queda significativa no atendimento de enfermagem no ambulatório em discussão, porém no ano de 2021 essa diferença foi reduzida.

Gráfico2 - Procedimentos de enfermagem realizados em 2019.

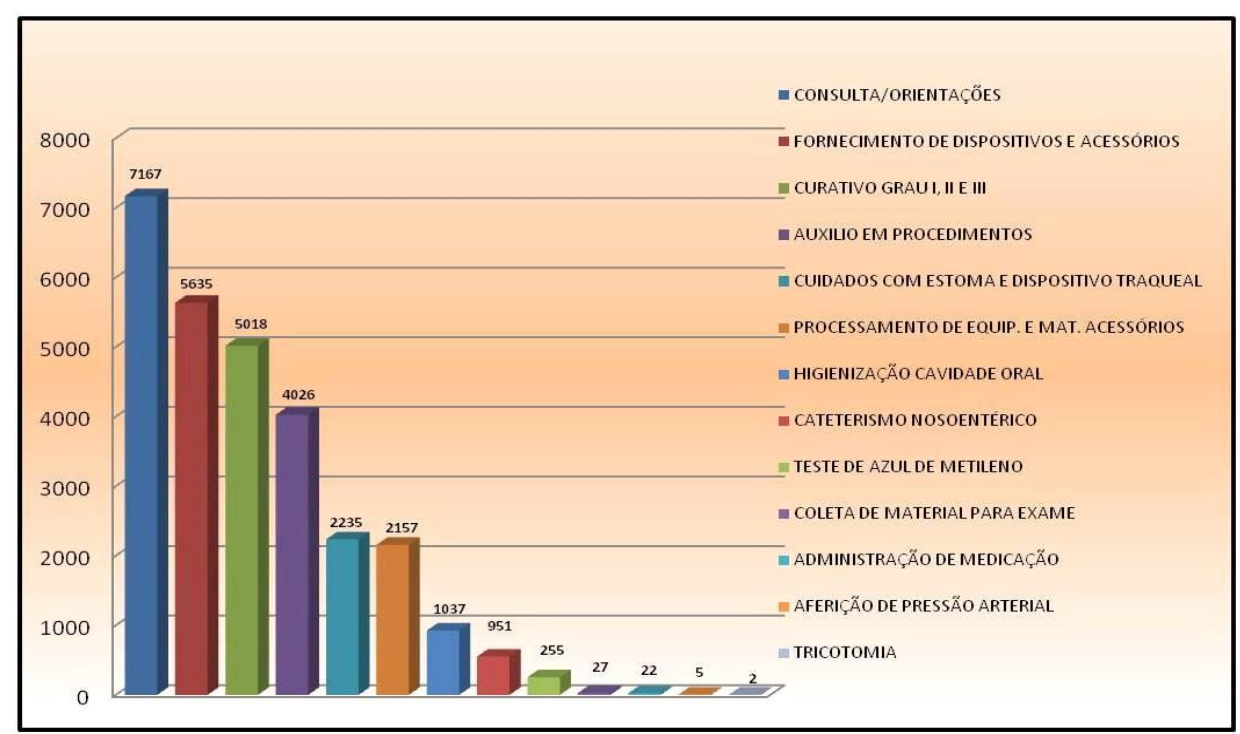

Fonte: INCA - HC1.

O Gráfico 2 apresenta os procedimentos mais habituais realizados pela equipe de enfermagem no ano de 2019, com destaque para: as consultas e orientações; o fornecimento de dispositivos e acessórios para continuidade do cuidado em domicílio e a realização dos curativos. É importante destacar que neste último procedimento, de acordo com o COFEN (2018), o enfermeiro é o profissional que coordena e supervisiona a equipe de enfermagem, bem como avalia, prescreve e executa cuidados aos pacientes com todos os tipos de feridas, independente do grau de comprometimento tecidual. Além disso, participa da escolha de materiais, equipamentos e medicamentos para prevenção e cuidados. 
Gráfico 3 - Procedimentos de enfermagem realizados em 2020.

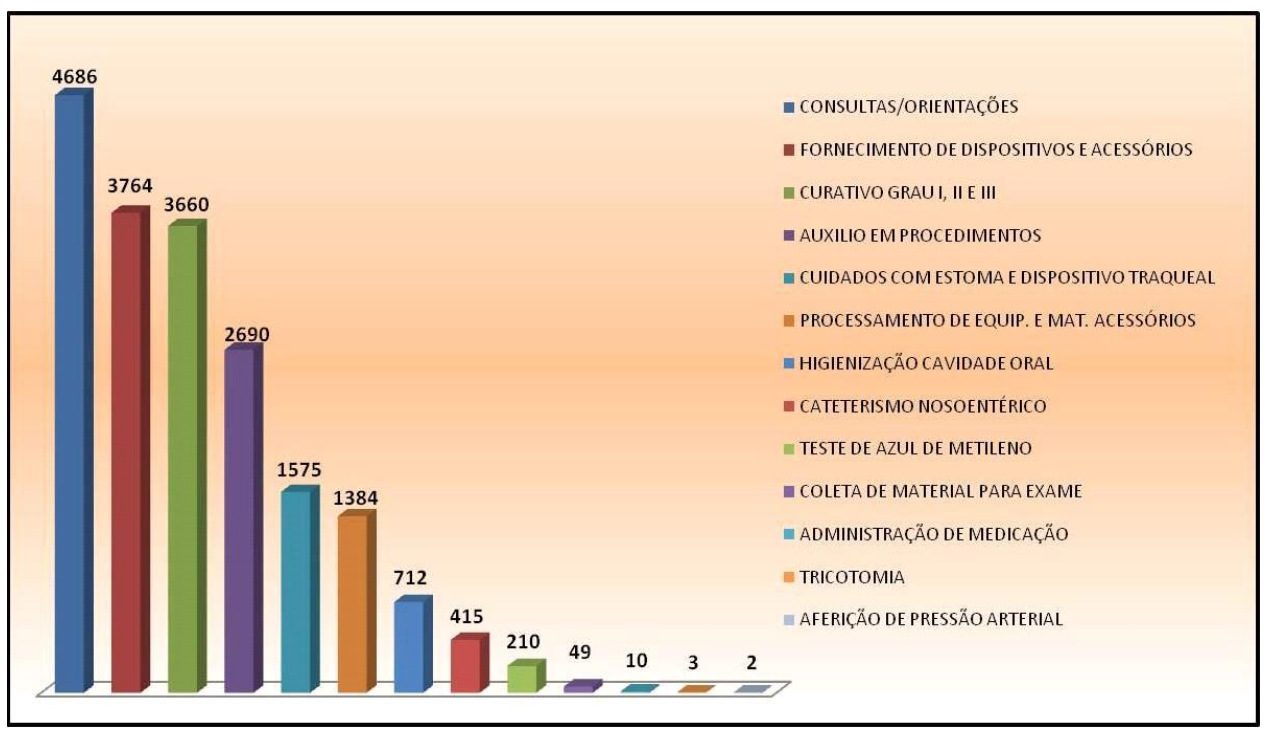

Fonte: INCA - HC1.

De acordo com o Gráfico 3, percebe-se que no ano de 2020 não houve mudança no que se refere aos procedimentos realizados pela enfermagem. Porém, ao observar o quantitativo dos procedimentos realizados, nota-se uma queda em números, bastante significativa, que vem corroborar com o declínio no número de pacientes atendidos. Sendo assim é possível notar que as orientações se mantiveram em primeiro lugar, já que este procedimento continuou acontecendo de forma presencial aos pacientes quando esses recebiam alta hospitalar e via telefone, quando solicitado.

Gráfico 4 - Procedimentos de enfermagem realizados em 2021.

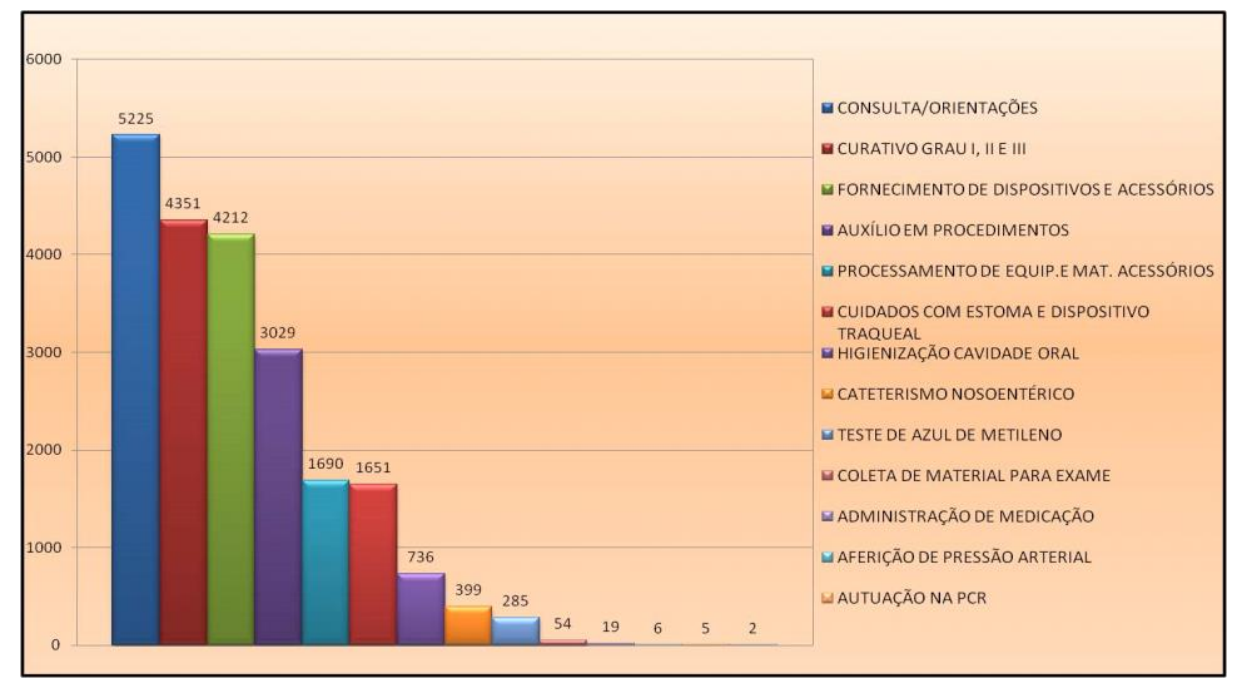

Fonte: INCA - HC1.

No Gráfico 4, percebe-se que houve uma mudança do ranque de procedimentos realizados pela equipe de enfermagem em relação ao ano de 2019 e 2020, onde a realização de curativos aparece em segundo lugar. Esse resultado se justifica pela adaptação e elevação do conhecimento dos gerentes e da equipe de enfermagem na qualidade das estratégias implementadas para prevenção da COVID-19, possibilitando o aumento das consultas médicas e de enfermagem, bem como a entrada dos acompanhantes na instituição, já que a presença do cuidador/familiar junto ao paciente, nas consultas de enfermagem, proporciona melhores condições para o cuidado em domicílio. 
Gráfico 5 - Procedimentos de enfermagem realizados no ambulatório de cirurgia de cabeça e pescoço no ano 2019,2020 e 2021.

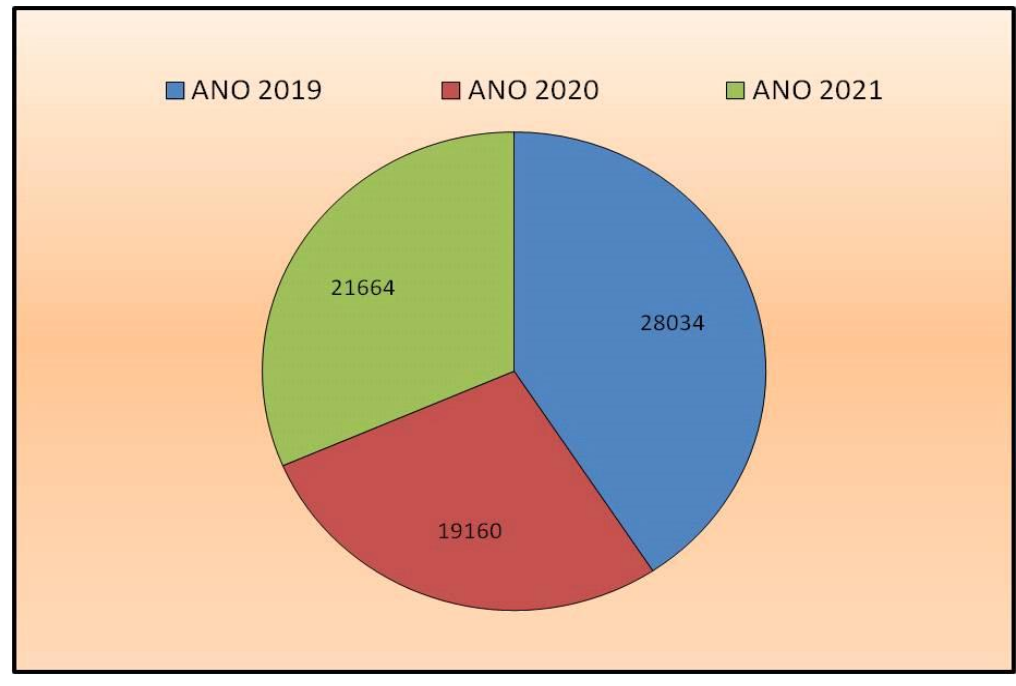

Fonte: INCA - HC1.

No que se refere ao quantitativo de procedimentos realizados em 2020 (Gráfico 5), foi possível observar que houve uma queda de $31 \%$ em relação a 2019 e, em comparação a 2021, houve uma queda de $26 \%$, porém comparando o ano de 2020 para 2021, houve um aumento de $13 \%$.

\section{Discussão}

A experiência de 20 anos de uma das autoras, enfermeira responsável pelo ambulatório de atendimento de pacientes com câncer de cabeça e pescoço, em um Hospital Público Federal, referência em oncologia no Rio de Janeiro, relata a alta demanda de cuidados específicos da equipe de Enfermagem e a necessidade de ofertar um atendimento integralizado em todas as fases assistenciais oncológicas, ou seja, desde a confirmação do diagnóstico até a reabilitação.

As ações de enfermagem prestadas aos pacientes neste ambulatório são inúmeras, conforme resultado apresentado nesse estudo, porém alguns são mais prevalentes, independente do período antes ou após a COVID-19, como: orientações, fornecimento de dispositivos e acessórios para a continuidade do cuidado em domicílio e o cuidado com as feridas tumorais e cirúrgicas, estomas respiratórios e trocas de cânulas de traqueostomia.

A equipe de enfermagem, bem como a equipe multidisciplinar, atua no atendimento dos pacientes de forma singular e qualificada, porém a pandemia trouxe dificuldades para o desenvolvimento das atividades de enfermagem ao paciente, tanto pela preocupação do mesmo em estar em um ambiente suscetível à contaminação por COVID-19, quanto aos profissionais na abordagem do paciente e familiar no referido ambulatório.

Devido à redução no agendamento dos pacientes para consultas de primeira vez e subsequente, conforme resultado apresentado no gráfico 1, os tratamentos clínicos e cirúrgicos foram comprometidos devido ao agravo da COVID-19 no ano de 2020, refletindo também no atendimento de enfermagem. Os pacientes não conseguiram acesso para realizar as sessões de radioterapia e quimioterapia. De acordo com Denys et al. (2020), em um estudo realizado com pacientes oncológicos, 43\% destes relataram ter seu tratamento impactado pela pandemia. Porém, no ano de 2021, aos poucos as consultas médicas e de enfermagem foram retornando a normalidade, pois como em outras instituições de saúde, o hospital foi se aprimorando nas estratégias de implementação de medidas de segurança para o enfrentamento da pandemia.

O tratamento para o câncer de cabeça e pescoço deriva de consultas de primeira vez, alguns tipos de terapêutica como a cirurgia e as radioterapias programadas foram canceladas ou adiadas devido à priorização de leitos hospitalares e de 
profissionais para aqueles que estavam gravemente doentes com infecção pela COVID-19, pois as literaturas científicas evidenciaram que essa infecção afeta os pacientes em tratamento para o câncer de forma mais severa (Araújo et al., 2020).

$\mathrm{O}$ cuidado da equipe de enfermagem com o paciente oncológico de cabeça e pescoço é bastante complexo devido ao grau de complicação relacionado ao tratamento, às condições clínicas, além das dificuldades cognitivas percebidas no decorrer das orientações fornecidas durante a consulta de enfermagem ou no momento da realização dos procedimentos pelos técnicos.

Dentre as ações desenvolvidas pela equipe de enfermagem, foi possível perceber nos resultados, que as orientações fornecidas aos pacientes e acompanhantes emergiu em primeiro lugar em todos os anos, independentemente da situação pandêmica do País, pois esse cuidado é necessário e de extrema relevância neste tipo de clientela, para evitar intercorrências, tais como: infecções nos sítios cirúrgicos, infestações por miíase no estoma respiratório ou na ferida tumoral, bem como para promover e incentivar o paciente a desenvolver o autocuidado (Origa \& Costa, 2020).

A enfermagem é uma das profissões da área da saúde cuja essência e especificidade destaca o cuidado com o ser humano, individualmente, na família ou na comunidade, desenvolvendo atividades distintas. Ela se responsabiliza pelo conforto, acolhimento e bem-estar dos pacientes, seja prestando o cuidado, seja coordenando outros setores para a prestação da assistência e promovendo a autonomia dos pacientes (Valença et al., 2016).

A realização de procedimentos, de acordo com as atribuições de cada componente da equipe de enfermagem, contribui para que haja uma interação entre os profissionais, como também um entendimento correto sobre os papéis que cada um executa na instituição, cooperando para que as atividades exercidas resultem numa assistência de enfermagem integralizada e de qualidade prestada aos clientes.

No enfrentamento de um diagnóstico de câncer e em especial na região de cabeça e pescoço, o paciente responde de modo individual as reações como: medo, ansiedade, tristeza, revolta pela alteração da imagem provocada pela lesão tumoral e a utilização da cânula de traqueostomia, que o impede de se expressar de forma verbal e se relacional socialmente.

Seno assim, o desafio da assistência de enfermagem prestada ao paciente de CP está nas suas varias demandas físicas e psicossociais, pelas quais necessitam ser atendidas por meios de diferentes formas de comunicação e aconselhamento, além de conhecimentos teóricos e práticos que envolvem o cuidado (Cruz et al., 2016).

Deste modo, é possível afirmar que o cenário pandêmico proporcionou danos na qualidade de vida dos pacientes com câncer de cabeça e pescoço, e que o maior impacto ocorreu na dimensão biológica, como resultado do risco aumentado de complicações associadas à COVID-19, como dificuldade de acesso ao tratamento (Corrêa et al., 2020).

O impedimento da entrada na Instituição do paciente com acompanhante tornou a comunicação mais difícil e ineficiente, já que as informações prestadas ao mesmo, para o cuidado de higiene das cânulas, limpeza dos estomas respiratórios, bem como do cuidado com as lesões tumorais ou feridas operatórias, faz-se necessária ser prestadas ao máximo de pessoas envolvidas no contexto de cuidado com o paciente de $\mathrm{CP}$, por entender que esse apresenta dificuldades para gerenciar todo o processo de cuidados relacionados à patologia (INCA, 2020).

$\mathrm{O}$ paciente de $\mathrm{CP}$ apresenta dificuldades de comunicação que podem ocorrer antes ou após a cirurgia, como: medo de realizar os cuidados de higiene dos estomas, da manipulação da cânula de traqueostomia, de manter higiene corporal que vem corroborar com os conceitos e postulados de Dorothea Orem sobre a construção do conhecimento no campo das bases teóricas e filosóficas da enfermagem, como forte norteador da prática da mesma, no que se refere às necessidades de autocuidado e na promoção de saúde por meio de intervenções educacionais (Santos \& Mota, 2021).

De acordo com a teoria de Maslow, quando o indivíduo atinge, seja total ou parcialmente as suas necessidades, consegue alcançar níveis mais elevados de saúde psicológicos, que está imbricado com a forma de como um ser reage às exigências, desafios e mudanças de vida e também com o modo que harmoniza suas ideias e emoções (Corrêa et al., 2020). 
Assim, entende-se que o enfermeiro deve agir de forma a orientar as ações desenvolvidas junto ao paciente, incluindo o cuidador/familiar, que deverá ser acolhido e incluído nas ações desenvolvidas junto ao indivíduo, participando ativamente em toda a fase do tratamento, assim como da prevenção dos agravos no ambiente domiciliar, pois o tratamento ou mesmo a patologia torna mais exaustivo o processo de comunicação com a equipe de enfermagem, que durante a pandemia ficou mais difícil e ineficaz.

O trabalho em equipe entendida como corpo técnico, mas também como grupo social, favorece o surgimento das estratégias de enfrentamento e suporte para a atuação profissional e fonte de resolução de problemas (Santos et al., 2016).

Os procedimentos técnicos reportados pela equipe de enfermagem em indivíduos com tumores na região de cabeça e pescoço nos apresentam uma compreensão da relação enfermagem-indivíduos antes, durante e após a alta hospitalar, devendo os achados serem considerados por enfermeiros que prestam informações para o cuidado domiciliar.

Desta forma, garantir a continuidade do tratamento para os pacientes no ambulatório de cirurgia de cabeça e pescoço, bem como em toda a instituição se tornou prioridade e um desafio no decorrer da evolução da pandemia da COVID-19, muitas recomendações técnicas e práticas foram elaboradas, apreendidas e implementadas (Araujo et al., 2020).

\section{Conclusão}

No ano de 2020, período de início da pandemia por COVID-19, as instituições de saúde, tendo que se habituar a nova forma de atendimento aos pacientes, tiveram que elaborar e implementar estratégias para adaptar o atendimento aos mesmos, suspendendo a circulação de acompanhantes, causando grande dificuldade para promover a assistência de enfermagem relacionada às orientações para a continuidade do cuidado em domicílio, apenas para o paciente, já que nesse contexto, o envolvimento do cuidador/familiar a essa clientela é de suma importância.

As estratégias também permearam no sentido de proteger os profissionais quanto à contaminação pelo vírus, mesmo assim houve um alto índice de absenteísmo no cenário de cuidado, prejudicando a qualidade dos serviços prestados.

A equipe de enfermagem, por estar mais próxima e por mais tempo junto ao paciente e seus familiares e ser responsável pela prestação de cuidados em geral precisa estar em boas condições físicas e psicológicas para perceber os pacientes e prestar uma assistência de qualidade, compreendendo-os em todas as suas necessidades e dimensões, no decorrer do processo de adoecimento.

As orientações de cuidados são consideradas essenciais na prevenção, promoção e na reabilitação da saúde do cliente. O desafio da assistência de enfermagem prestada a essa população está nas suas várias demandas físicas e psicossociais, as quais precisam ser atendidas por meio de diferentes formas de comunicação e aconselhamento, além de conhecimentos teóricos e práticos especializados, que envolvam o cuidado.

Desta forma, conclui-se que a pandemia por COVID-19 gerou um forte impacto na assistência prestada aos pacientes do ambulatório de cirurgia de cabeça e pescoço, levando a uma redução do atendimento e da prestação dos cuidados de enfermagem.

Sugere-se assim, que mais estudos sobre a temática sejam realizados para tornar conhecidas as ações desenvolvidas pela equipe de enfermagem no cenário oncológico de outras instituições.

\section{Referências}

Araujo, S. E. A., Leal, A., Centrone, A. F. Y., Teich, V. D., Malheiro, D. T., Cypriano, A. S., Cendoroglo Neto, M. \& Klajner, S. (2020) Impacto da COVID19 sobre o atendimento de pacientes oncológicos: experiência de um centro oncológico localizado em um epicentro Latino-Americano da pandemia. Jornal Einstein. https://journal.einstein.br.

Bardin, L. Análise de conteúdo. Edições 70, 2004. 
Research, Society and Development, v. 11, n. 3, e17311326321, 2022

(CC BY 4.0) | ISSN 2525-3409 | DOI: http://dx.doi.org/10.33448/rsd-v11i3.26321

Brasil (2019) Instituto Nacional de Câncer José Alencar Gomes da Silva. Estimativa 2020: incidência de câncer no Brasil / Ministério da Saúde /Instituto Nacional de Câncer José Alencar Gomes da Silva. INCA.

Conselho Federal de Enfermagem - COFEN (2018) Anexo da Resolução Cofen $n^{o}$ 0567/2018. Regulamento da atuação da equipe de enfermagem no cuidado aos pacientes com feridas.

Corrêa, K. M., Oliveira, J. D. B. \& Taets, G. G. C. C. (2020) Impacto na Qualidade de Vida de Pacientes com Câncer em meio à Pandemia de Covid-19: uma Reflexão a partir da Teoria das Necessidades Humanas Básicas de Abraham Maslow. RBC Revista Brasileira de Cancerologia. 66. https://doi.org/10.32635/2176-9745.RBC.2020v66nTemaAtual.1068v.66 n.TemaAtual

Cruz, F. O. A. M., Ferreira, E. B., Vasques, C. I., Mata, L. R. F. \& Reis, P. E. D (2016) Validação de manual educativo para pacientes com câncer de cabeça e pescoço submetidos à radioterapia. Rev. latino-am. Enfermagem. V.24. https://doi.org/10.1590/1518-8345.0949.2706

Denys, A., Guiu, B., Chevalier, P., Digklia, A., Kerviler, E. \& Baere T. (2020) Interventional oncology at the time of COVID-19 pandemic: Problems and solutions. Diagnostic and Interventional Imaging, 101(6), 347-353. https://doi 10.1016/j.diii.2020.04.005.

Instituto Nacional de Câncer - INCA (2020) INCA contra o coronavirus. https://www.inca.gov.br/noticias/inca-contra-coronavirus

Lagergren, J. Andersson,G., Talbäck, M., Drefahl, S., Bihagen, E. , Härkönen, J., Feychting, M. \& Ljung, R. (2016) Status, education, and income in relation to the risk of esophageal and gastric cancer by histological type and site. Cancer, 122 (2): 207-12. https://doi.org/10.1002/cncr.29731.

Ramos, R. S. A. (2020) Oncology Nursing in Copingwith the COVID-19 Pandemic: Reflections and Recommendations for Oncology Care Practice Enfermería em Oncología para hacer frente a la Pandemia Covid-19. RBC Revista Brasileira de Cancerologia. https://doi.org/10.32635/21769745.RBC.2020v66nTemaAtual.1007.

Mota, L. P., Carvalho, M. R. M. A., Carvalho Neto, A. L. Ferreira, F. A. A., Poty, J. A. C., Pompeu, J. G. F., Rocha, M. M. L. R., Fé, R. C. M., Silva, L. E. B, Carvalho Neto, E. A., Silva, F. M. S., Oliveira, C. P. C., Rabelo, M. N., Marques, L. L. B. L., Oliveira, M. G. S. \& Cruz, I. V. (2021) Neoplasia de cabeça e pescoço: principais causas e tratamentos. RSD Research Society and Development. 10(5). https://doi.org/10.33448/rsd-v10i5.15113

Origa, V. C. M. \& Costa, E. A. (2020) A dimensão educativa da enfermagem no seu processo de trabalho. Revista Científica Multidisciplinar Núcleo do Conhecimento. 04, 95-133. https://www.nucleodoconhecimento.com.br/saude

Santos, N. A. R., Santos, A. T. C \& Silva, R. P. (2016) Estratégias de enfrentamento de enfermeiros no cuidado aos pacientes com neoplasias de cabeça e pescoço, Rev Esc Enferm USP. 2016;50(4):569-578. https://www.scielo.br/j/reeusp/a/7NYcHfBtq3kGq8MtfGBPzGN/?lang=en.

Santos, M. J. \& Mota, L. M. (2021) O uso da teoria de orem no cuidado em pacientes com hipertensão arterial sistêmica na atenção básica, unit. Universidade tiradente. OpenRIT - Repositório Institucional Tiradentes. http://openrit.grupotiradentes.com:8080/xmlui/handle/set/3756/dimensao-educativa.

Valença, C. N., Silva, A. F. C., Marinho, C. S. R., Silva, M. L. P., Sousa, Y. G.\& Medeiros, S. M. (2016) Vivências dos profissionais da enfermagem sobre procedimentos executados no hospital. Revista Cubana de Enfermería. 32(4). http://www.revenfermeria.sld.cu/index.php/enf/article/view/1010.

World Health Organization (WHO) (2020) Coronavirus disease (COVID - 2019) situation reports WHO. 